EPJ Web of Conferences 20, 01007 (2012)

DOI: $10.1051 /$ epjconf/20122001007

(C) Owned by the authors, published by EDP Sciences, 2012

\title{
Quark models for mesons
}

\author{
Jialun Ping ${ }^{1, a}$, Chengrong Deng ${ }^{2}$, Hongxia Huang ${ }^{1}$, Fangfang Dong ${ }^{1}$, Fan Wang ${ }^{3}$ \\ ${ }^{1}$ Department of Physics, Nanjing Normal University, Nanjing 210097, P.R. China \\ ${ }^{2}$ School of Mathematics and Physics, Chongqing Jiaotong University, Chongqing 400074, P.R. China \\ ${ }^{3}$ Department of Physics, Nanjing University, Nanjing 210093, P.R. China
}

\begin{abstract}
With the advance of experiment, many high excited states of meson are identified. It is found that many of them are difficult to fit into conventional $q \bar{q}$ meson spectra. Various explanations are proposed. One of them is the four-quark states or molecules of two mesons. In the framework of quark model, by taking into account of multi-body interaction, four-quark states are studied. The results show that the scalar mesons: $f_{0}(600), f_{0}(980)$ and $Y(2175)$ can be well described by four-quark states. Furthermore, unquenched quark model, which incorporates the four-quark components in the conventional $q \bar{q}$ states, is constructed for mesons. By readjusting the model parameters, the model can give a good description of mesons. The model is also used to study the newly discovered $X Y Z$ particles.
\end{abstract}

\section{Introduction}

Hadrons (meson and baryon) are the strong interacting systems, which are the main source we get the information of quantum chromodynamics (QCD), the fundamental theory of strong interaction. Because of the complexity and the non-perturbative property of QCD in low-energy region, To get a reliable results for hadrons is still a challenge task for QCD. Although lattice QCD, QCD sumrule, Dyson-schwinger approach and other non-perturbative method make some impressive progresses recently, the QCD-inspired quark model is still a powerful and common used method to study the properties of hadrons.

In quark models, the naive picture of meson $(q \bar{q})$ and baryon $(q q q)$ achieved a tremendous success on the properties of hadrons due to the unique color structure of hadrons. However it also limits us to obtain the more information on color properties of QCD. Multiquark system is indispensable to explore the various color structure of QCD [1]. In fact, there are many high Fock components in a meson state in addition to $q \bar{q}$,

$$
|M\rangle=|q \bar{q}\rangle+|q \bar{q} q \bar{q}\rangle+|q \bar{q} g\rangle+\left|q^{3} \bar{q}^{3}\right\rangle+\cdots
$$

In naive quark model, the effects of high Fock states are renormalized into the parameters. In most case, it is a good approximation, especially for ground state hadrons with heavy quarks. Recently, BaBar, Belle and other collaborations discovered some new charmonium-like states ("XYZ" states) above the open charm threshold, which is difficult to fit into the conventional pictures of $c \bar{c}$ mesons [2]. A lot of explanations are proposed for "XYZ" states, tetraquark states, molecular states, hybrid, charmonium, etc. [2].

To unify the description of mesons, the construction of unquenched quark model is needed. In the unquenched approach, the high Fock components are taken into account. The present work is trying to study the charmonium spectrum by including the four-quark component in addition to $q \bar{q}$ in the calculation.

a e-mail: jlping@njnu.edu.cn

This is an Open Access article distributed under the terms of the Creative Commons Attribution-Noncommercial License 3.0, which permits unrestricted use, distribution, and reproduction in any noncommercial medium, provided the original work is properly cited. 


\section{EPJ Web of Conferences}

The structure of the paper is as follows, Sect. 2 gives a brief introduction of the conventional quark model, the chiral quark model. A string-like quark model is introduce in Sect. 3 and apply to fourquark system. The unquenched quark model for $c \bar{c}$ is given in Sect.4. Finally a summary is given in the last section.

\section{Constituent Quark Models}

The important properties of QCD in the low-energy region are color confinement and the spontaneous breaking of chiral symmetry. The spontaneous breaking of chiral symmetry leads to that the quarks obtain their constituent masses and interact with each other through the exchange of Goldstone bosons [3]. The color confinement is imitated by introducing color confinement potential and the perturbative part of QCD is retained by effective one gluon exchange in quark model. Generally the chiral partner $\sigma$-meson is invoked to account for the intermediate-range attraction of nucleon-nucleon interaction in the chiral quark model and the chiral quark gives a good description of meson spectrum [4]. However, there are some controversies on the use of $\sigma$-meson and it is argued recently that the $\sigma$ meson exchange used in the chiral quark model can be replaced by the quark delocalization and color screening mechanism [5]. In the following we show that the quark model without $\sigma$-meson exchange can also describe the meson spectrum well. The model Hamiltonian of the $q \bar{q}$ system $(n=2)$ is

$$
\begin{aligned}
H & =\sum_{i=1}^{n}\left(m_{i}+\frac{\mathbf{p}_{i}^{2}}{2 m_{i}}\right)-T_{C M}+\sum_{i>j}^{n}\left(V_{i j}^{C}+V_{i j}^{G}+V_{i j}^{\chi}\right), \quad \chi=\pi, K, \eta \\
V_{i j}^{\pi} & =\frac{g_{c h}^{2}}{4 \pi} \frac{m_{\pi}^{2}}{12 m_{i} m_{j}} \frac{\Lambda_{\pi}^{2}}{\Lambda_{\pi}^{2}-m_{\pi}^{2}} m_{\pi} \sigma_{i} \cdot \sigma_{j}\left[Y\left(m_{\pi} r_{i j}\right)-\frac{\Lambda_{\pi}^{3}}{m_{\pi}^{3}} Y\left(\Lambda_{\pi} r_{i j}\right)\right] \sum_{a=1}^{3} F_{i}^{a} \cdot F_{j}^{a} \\
V_{i j}^{K} & =\frac{g_{c h}^{2}}{4 \pi} \frac{m_{K}^{2}}{12 m_{i} m_{j}} \frac{\Lambda_{K}^{2}}{\Lambda_{K}^{2}-m_{\pi}^{2}} m_{K} \sigma_{i} \cdot \sigma_{j}\left[Y\left(m_{K} r_{i j}\right)-\frac{\Lambda_{K}^{3}}{m_{K}^{3}} Y\left(\Lambda_{K} r_{i j}\right)\right] \sum_{a=4}^{7} F_{i}^{a} \cdot F_{j}^{a} \\
V_{i j}^{\eta} & =\frac{g_{c h}^{2}}{4 \pi} \frac{m_{\eta}^{2}}{12 m_{i} m_{j}} \frac{\Lambda_{\eta}^{2}}{\Lambda_{\eta}^{2}-m_{\eta}^{2}} m_{\eta} \sigma_{i} \cdot \sigma_{j}\left[Y\left(m_{\eta} r_{i j}\right)-\frac{\Lambda_{\eta}^{3}}{m_{\eta}^{3}} Y\left(\Lambda_{\eta} r_{i j}\right)\right]\left[\cos \theta_{P}\left(F_{i}^{8} \cdot F_{j}^{8}\right)-\sin \theta_{P}\right], \\
V_{i j}^{G} & =\frac{1}{4} \alpha_{s} \lambda_{i}^{c} \cdot \lambda_{j}^{c}\left[\frac{1}{r_{i j}}-\frac{\pi}{2} \delta\left(\mathbf{r}_{i j}\right)\left(\frac{1}{m_{i}^{2}}+\frac{1}{m_{j}^{2}}+\frac{4}{3 m_{i} m_{j}} \sigma_{i} \cdot \sigma_{j}\right)\right], \\
V_{i j}^{C} & =k\left(r_{i j}^{2}-\Delta\right) .
\end{aligned}
$$

where $T_{C M}$ is the center-of-mass kinetic energy, $Y(x)$ is the standard Yukawa function and all symbols have their usual meaning. The delta function in the one-gluon exchange potential should be regularized because of the finite size of the constituent quark [6], the regularization is flavor dependent [4,7],

$$
\delta\left(\mathbf{r}_{i j}\right)=\frac{1}{4 \pi} \frac{1}{r_{i j} r_{0}^{2}(\mu)} e^{-r_{i j} / r_{0}(\mu)},
$$

where $\mu$ is the reduced mass of the $q \bar{q}$ system and $r_{0}(\mu)=\hat{r}_{0} / \mu$ and the $\hat{r}_{0}$ is a model parameter.

The model parameters are fixed as follows: the $u, d$-quark masses are taken as the same and are assumed to be exactly $\frac{1}{3}$ of the nucleon mass, namely $m_{u}=m_{d}=313 \mathrm{MeV}$, the masses of $\pi, K, \eta$ take the experimental values, the $\Lambda_{\pi}, \Lambda_{K}, \Lambda_{\eta}, \theta_{p}, \Lambda_{0}$ and $\mu_{0}$ are taken the same values as in Ref.[4], namely $\Lambda_{\pi}=4.2 \mathrm{fm}^{-1}, \Lambda_{K}=\Lambda_{\eta}=5.2 \mathrm{fm}^{-1}, \theta_{p}=-15^{\circ}, \Lambda_{0}=36.98 \mathrm{MeV}$ and $\mu_{0}=0.113 \mathrm{fm}$. The chiral coupling constant $g_{c h}$ is determined from the $\pi N N$ coupling constant through

$$
\frac{g_{c h}^{2}}{4 \pi}=\left(\frac{3}{5}\right)^{2} \frac{g_{\pi N N}^{2}}{4 \pi} \frac{m_{u, d}^{2}}{m_{N}^{2}}
$$


and flavor $S U(3)$ symmetry is assumed, where $\frac{g_{c h}^{2}}{4 \pi}=0.54$. The rest parameters $m_{s}, k, \Delta, \alpha_{0}$ and $\hat{r}_{0}$ are determined by fitting ground state meson spectra. An effective scale-dependent strong coupling constant [4] is given by

$$
\alpha_{s}(\mu)=\frac{\alpha_{0}}{\ln \left[\frac{\mu^{2}+\mu_{0}^{2}}{\Lambda_{0}^{2}}\right]} .
$$

The meson spectra are obtained by solving the following Schrödinger equation

$$
\left[-\frac{\hbar^{2}}{2 \mu} \nabla^{2}+V(\mathbf{r})-E\right] \psi_{l m}(\mathbf{r}) \psi_{c s f}=0
$$

where $\psi_{l m}$ is the relative orbital motion wave functions, $\psi_{c s f}$ is the color-spin-flavor wave function. $\psi_{l m}(\mathbf{r})$ can be expanded by means of Gaussian functions with different size [8]

$$
\phi_{l m}(\mathbf{r})=\sum_{n=1}^{n_{\max }} c_{n} N_{n l} r^{l} e^{-v_{n} r^{2}} Y_{l m}(\hat{\mathbf{r}}) .
$$

Gaussian size parameters are taken as geometric progression

$$
v_{n}=\frac{1}{r_{n}^{2}}, r_{n}=r_{1} a^{n-1}, a=\left(\frac{r_{n_{\max }}}{r_{1}}\right)^{\frac{1}{n_{\max }-1}} .
$$

With $r_{1}=0.1 \mathrm{fm}, r_{n_{\max }}=2.0 \mathrm{fm}$ and $n_{\max }=7$, we can obtain converged results. The model parameters

\begin{tabular}{|c|c|c|c|c|c|c|}
\hline $\begin{array}{c}m_{s} \\
(\mathrm{MeV}) \\
520\end{array}$ & $\begin{array}{c}m_{c} \\
(\mathrm{MeV})\end{array}$ & $\begin{array}{c}k \\
\left(\mathrm{MeV} \mathrm{fm}^{-2}\right)\end{array}$ & & $\begin{array}{c}\hat{r}_{0} \\
(\mathrm{MeV} \mathrm{fm})\end{array}$ & $\begin{array}{c}\Delta \\
\left(\mathrm{fm}^{2}\right) \\
0.5\end{array}$ & 4.25 \\
\hline \multicolumn{7}{|c|}{ Table 2. Meson spectra $(\mathrm{MeV})$. } \\
\hline Meson: & $\pi$ & $\mathrm{K}$ & $\rho$ & $K^{*}$ & $\omega$ & $\phi$ \\
\hline Theo. $(L=0)$ & 139 & 502 & 761 & 897 & 735 & 1023 \\
\hline Exp. $(L=0)$ & 139 & 496 & 770 & 898 & 780 & 1020 \\
\hline Theo. $(L=1)$ & 1054 & 1204 & 1102 & 1226 & 1098 & 1342 \\
\hline Meson: & $D^{0}$ & $D^{*}$ & $D_{s}$ & $D_{s}^{*}$ & $\eta_{c}$ & $J / \psi$ \\
\hline Theo. $(L=0)$ & 1928 & 2001 & 2014 & 2112 & 2992 & 3144 \\
\hline Exp. $(L=0)$ & 1865 & 2007 & 1968 & 2112 & 2980 & 3097 \\
\hline Theo. $(L=1)$ & 2304 & 2310 & 2401 & 2405 & 3419 & 3420 \\
\hline
\end{tabular}
and part of the obtained meson masses are shown in the Table 1 and 2, respectively. The more detailed results can be seen in Ref.[9].

Table 1. The model parameters.

From Table 2, one can see that the calculated meson masses are consistent with experimental values. The masses of mesons with orbital angular momentum $L=1$ are also given in Table 2 . As in other work, the low-lying scalar mesons, $f_{0}(600), f_{0}(980), \cdots$ and most newly observed states $Y(2175), X(3872), \cdots$, can not be described well in the model. A possible reason is that those states are tetraquark states, which we present in the following section.

\section{The String model for tetraquark states}

In multiquark system, the interaction among quarks is genuinely a multi-body interaction. The recent calculation of lattice QCD on the two-, three-, four- and five-quark systems show that the interactions 
among quarks can be approximated by the following formula [10],

$$
\begin{aligned}
V_{q \bar{q}} & =-A_{q \bar{q}} \frac{1}{\left|\mathbf{r}_{q}-\mathbf{r}_{\bar{q}}\right|}+\sigma_{q \bar{q}}\left|\mathbf{r}_{q}-\mathbf{r}_{\bar{q}}\right|+C_{q \bar{q}}, \\
V_{q^{3}} & =-A_{q^{3}} \sum_{i>j} \frac{1}{\left|\mathbf{r}_{i}-\mathbf{r}_{j}\right|}+\sigma_{q^{3}} L_{\min }+C_{q^{3}}, \\
V_{q^{2} \bar{q}^{2}} & =-A_{q^{2} \bar{q}^{2}} \sum_{i>j} \frac{1}{\left|\mathbf{r}_{i}-\mathbf{r}_{j}\right|}+\sigma_{q^{2} \bar{q}^{2}} L_{\min }+C_{q^{2} \bar{q}^{2}}, \\
V_{q^{4} \bar{q}} & =-A_{q^{4} \bar{q}} \sum_{i>j} \frac{1}{\left|\mathbf{r}_{i}-\mathbf{r}_{j}\right|}+\sigma_{q^{4} \bar{q}} L_{\min }+C_{q^{4} \bar{q}}, \\
L_{\min } & =\sum_{i} L_{i}
\end{aligned}
$$

where $L_{i}$ is the length of the i-th string which connecting quarks and/or junctions. The universality of the "confinement" is shown by

$$
\sigma_{q \bar{q}}=\sigma_{q^{3}}=\sigma_{q^{2} \bar{q}^{2}}=\sigma_{q^{4} \bar{q}}
$$

The Casimir scaling in baryon and meson is justified by $A_{q \bar{q}}=2 A_{q^{3}}$.

Based on these calculations, a string (flux-tube) model is constructed [1]. For four-quark system, diquark-anti-diquark picture is often used because of the attractive property of diquark [11-14]. Here the picture is used to study the tetraquark state in the flux-tube model. The configuration is shown in Fig.1, where a quark (antiquark) is represented by a solid (hollow) dot, $\mathbf{r}_{i}$ is (anti)quark's position, $\mathbf{y}_{i}$ is the junction where three strings (flux tubes) meet. A thin line connecting a quark and a junction represents a fundamental representation, i.e. color triplet, and a thick line connecting two junctions is for a color sextet or other representations, namely a compound string. The different types of string may have different stiffness $[15,16]$. The overall color singlet is satisfied by the coupling $\left[[q q]_{\mathbf{3}}[\bar{q} \bar{q}]_{3}\right]_{\mathbf{1}}$ and $\left[[q q]_{6}[\bar{q} \bar{q}]_{\mathbf{6}}\right]_{1}$, subscripts represent the dimensions of color representations.

In the flux-tube model, the confinement potential of the tetraquark state takes the following form (To simplify the calculation, the length of the string is replaced by the square of the length),

$$
V^{C}=k\left[\left(\mathbf{r}_{1}-\mathbf{y}_{1}\right)^{2}+\left(\mathbf{r}_{2}-\mathbf{y}_{1}\right)^{2}+\left(\mathbf{r}_{3}-\mathbf{y}_{2}\right)^{2}\left(\mathbf{r}_{4}-\mathbf{y}_{2}\right)^{2}+\kappa_{d}\left(\mathbf{y}_{1}-\mathbf{y}_{2}\right)^{2}\right]
$$

where $k$ is the stiffness of the string with the fundamental representation $\mathbf{3}$ and $k \kappa_{d}$ is the compound string stiffness, which can be obtained by [16]

$$
\kappa_{\mathbf{d}}=\frac{C_{\mathbf{d}}}{C_{\mathbf{3}}}
$$

where $C_{\mathbf{d}}$ is the eigenvalue of the Casimir operator associated with the $S U(3)$ color representation $\mathbf{d}$ of the string, $C_{3}=\frac{4}{3}, C_{6}=\frac{10}{3}$ and $C_{8}=3$.

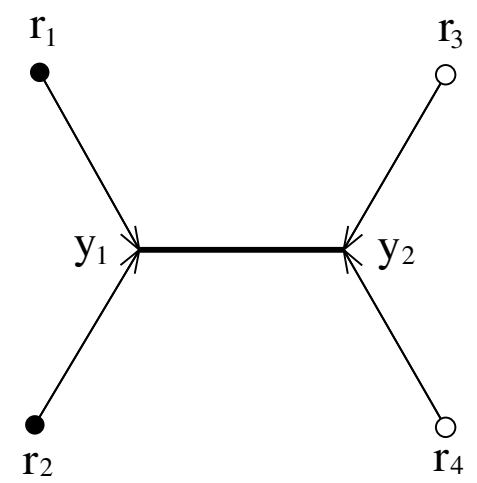

Fig. 1. Diquark-antidiquark state. 
For the given $\mathbf{r}_{i}$ 's, the $\mathbf{y}_{i}$ 's are fixed by minimizing the energy of the system. With fixed $\mathbf{y}_{i}$ 's and in the center of mass coordinate, the potential reduces to

$$
V^{C}=\frac{k}{m}\left(\mathbf{Q}_{1}^{2}+\mathbf{Q}_{2}^{2}+\frac{\kappa_{d}}{1+\kappa_{d}} \mathbf{Q}_{3}^{2}\right)
$$

where the canonical coordinates take the following form

$$
\begin{aligned}
& \mathbf{Q}_{1}=\sqrt{\frac{m}{2}}\left(\mathbf{r}_{1}-\mathbf{r}_{2}\right), \\
& \mathbf{Q}_{2}=\sqrt{\frac{m}{2}}\left(\mathbf{r}_{3}-\mathbf{r}_{4}\right), \\
& \mathbf{Q}_{3}=\sqrt{\frac{m}{4}}\left(\mathbf{r}_{1}+\mathbf{r}_{2}-\mathbf{r}_{3}-\mathbf{r}_{4}\right), \\
& \mathbf{Q}_{4}=\sqrt{\frac{m}{4}}\left(\mathbf{r}_{1}+\mathbf{r}_{2}+\mathbf{r}_{3}+\mathbf{r}_{4}\right) .
\end{aligned}
$$

if all quarks have the same mass. Taking into account of the potential energy shift (see Eq.(7) above), the confinement potential $V^{C}$ used in the present calculation has the following form

$$
V^{C}=k\left[\left(\frac{\left(\mathbf{r}_{1}-\mathbf{r}_{2}\right)^{2}}{2}-\Delta\right)+\left(\frac{\left(\mathbf{r}_{3}-\mathbf{r}_{4}\right)^{2}}{2}-\Delta\right)+\frac{\kappa_{d}}{1+\kappa_{d}}\left(\left(\frac{\mathbf{r}_{1}+\mathbf{r}_{2}}{2}-\frac{\mathbf{r}_{3}+\mathbf{r}_{4}}{2}\right)^{2}-\Delta\right)\right]
$$

Clearly our confinement potential is a multi-body interaction. The Hamiltonian for the four-quark system has the same as Eq.(2) with $n=4$ and replacing the confinement potential by Eq.(24).

The wave function of the four-quark system is

$$
\Phi_{I J_{T} M_{T}}=\left[\left[\left[\phi_{l_{1} m_{1}}^{G}(\mathbf{r}) \Psi_{s_{1} m_{s_{1}}}\right]_{J_{1} M_{1}}\left[\psi_{l_{2} m_{2}}^{G}(\mathbf{R}) \Psi_{s_{2} m_{s_{2}}}\right]_{J_{2} M_{2}}\right]_{J_{12} M_{12}} \chi_{L M}^{G}(\mathbf{X})\right]_{J_{T} M_{T}}\left[\Psi_{c_{1}} \Psi_{c_{2}}\right]_{c}\left[\Psi_{I_{1}} \Psi_{I_{2}}\right]_{I},
$$

where $I_{i}, s_{i}$ and $c_{i}, i=1,2$ represent isospin, spin and color of diquark and anti-diquark, respectively. The Jacobi coordinates are defined as

$$
\mathbf{r}=\mathbf{r}_{1}-\mathbf{r}_{2}, \quad \mathbf{R}=\mathbf{r}_{3}-\mathbf{r}_{4}, \quad \mathbf{X}=\frac{\mathbf{r}_{1}+\mathbf{r}_{2}}{2}-\frac{\mathbf{r}_{3}+\mathbf{r}_{4}}{2}
$$

The four-quark state is an overall color singlet with well defined parity $P=(-1)^{l_{1}+l_{2}+L}$, isospin $I$ and total angular momentum $J_{T}$.

The energy of the tetraquark states can be obtained by solving the four-body Schrödinger equation

$$
(H-E) \Phi_{I J_{T} M_{T}}=0 .
$$

To obtain a reliable solution of few-body problem, a high precision method is indispensable. The Gaussian Expansion Method (GEM) [8], which has been proven to be powerful in nuclear and hadron physics, is employed here. In GEM, three relative motion wave functions are written as,

$$
\begin{gathered}
\phi_{l_{1} m_{1}}^{G}(\mathbf{r})=\sum_{n_{1}=1}^{n_{1 \max }} c_{n_{1}} N_{n_{1} l_{1}} r^{l_{1}} e^{-v_{n_{1}} r^{2}} Y_{l_{1} m_{1}}(\hat{\mathbf{r}}) \\
\psi_{l_{2} m_{2}}^{G}(\mathbf{R})=\sum_{n_{2}=1}^{n_{2 \max }} c_{n_{2}} N_{n_{2} l_{2}} R^{l_{2}} e^{-v_{n_{2}} R^{2}} Y_{l_{2} m_{2}}(\hat{\mathbf{R}})
\end{gathered}
$$




$$
\chi_{L M}^{G}(\mathbf{X})=\sum_{n_{3}=1}^{n_{3 \max }} c_{n_{3}} N_{L M} X^{L} e^{-v_{n_{3}} X^{2}} Y_{L M}(\hat{\mathbf{X}})
$$

The choice of Gaussian size is the same as that of mesons (see Eq.(13). In GEM the calculated results are converged with $n_{1 \max }=10, n_{2 \max }=10$ and $n_{3 \max }=10$. Minimum and maximum ranges of the bases are $0.1 \mathrm{fm}$ and $3.0 \mathrm{fm}$ for coordinates $\mathbf{r}, \mathbf{R}$ and $\mathbf{X}$, respectively.

The model is applied to study the tetraquark states: $n \bar{n} n \bar{n}, n=u, d, s \bar{s} s \bar{s}$, etc. Part of the results are shown in Table 3. In obtaining the results, all the possible channels are included. So the model space is generally constructed by several thousands basis functions, for example, $Y(2175), I^{G} J^{P C}=0^{+} 1^{--}$, the number of basis functions is 2000 .

Table 3. Energies for some tetraquark states (MeV). $n=u, d$.

\begin{tabular}{ccccccc}
\hline \hline States & $n n \bar{n} \bar{n}$ & $n n \bar{n} \bar{n}$ & $n s \bar{n} \bar{s}$ & $n s \bar{n} \bar{s}$ & $s s \bar{s} \bar{s}$ & $s s \bar{s} \bar{s}$ \\
$I^{G} J^{P C}$ & $0^{+} 0^{++}$ & $0^{+} 0^{++}$ & $1^{-} 0^{++}$ & $1^{-} 1^{--}$ & $0^{+} 0^{++}$ & $0^{+} 1^{--}$ \\
Energy & 587 & 1019 & 1306 & 1715 & 1925 & 2176 \\
Candidate & $f_{0}(600)$ & $f_{0}(980)$ & $a_{0}(1450)$ & $X(1576)$ & $f_{0}(2020)$ & $Y(2175)$ \\
\hline States & $c n \bar{c} \bar{n}$ & $c n \bar{c} \bar{n}$ & $c n \bar{c} \bar{n}$ & $c n \bar{c} \bar{n}$ & $c s \bar{c} \bar{s}$ & $c s \bar{c} \bar{s}$ \\
$I^{G} J^{P C}$ & $0^{+} 1^{+-}$ & $0^{+} 0^{-+}$ & $0^{+} 0^{++}$ & $0^{+} 1^{--}$ & $0^{+} 0^{++}$ & $0^{+} 1^{--}$ \\
Energy & 3776 & 4020 & 3644 & 3978 & 4038 & 4292 \\
Candidate & & $X(3872)$ & & $Y(4008)$ & & $Y(4260)$ \\
\hline \hline
\end{tabular}

From the Table 3, some interesting results are worth to mention. For non-strange tetraquark, the masses of ground state and the first radial excited state with quantum numbers $I^{G} J^{P C}=0^{+} 0^{++}$are 587 $\mathrm{MeV}$ and $1019 \mathrm{MeV}$, which are very close to the experimental values of $f_{0}(600)$ and $f_{0}(980)$. So the scalar meson $f_{0}(600)$ and $f_{0}(980)$ can be identified as tetraquark states in the present calculation, other than the $q \bar{q} P$-wave exited states (see Table 2 ). The same conclusion is also obtained in the study of the lowest-lying scalar mesons by the QCD sum rule [17]. Another interesting state is $Y(2175)$, which is observed by Babar Collaboration near the threshold in the process $e^{+} e^{-} \rightarrow \phi f_{0}(980)$ via initialstate radiation [18] and is confirmed by BES collaboration in the process $J / \psi \rightarrow \eta \phi f_{0}(980)$ [19]. The Breit-Wigner mass is $M=2.175 \pm 0.010 \pm 0.015 \mathrm{GeV}$, and width is narrow $\Gamma=0.058 \pm 0.016 \pm$ $0.020 \mathrm{GeV}$. The quantum numbers are claimed as $I^{G} J^{P C}=0^{+} 1^{--}$. Various theoretical interpretations have been proposed to explain this resonance, strangeonium hybrid [20]. tetraquark state $s s \bar{s} \bar{s}$ [21], Swave threshold effects [22], $\phi K K$ system [23], etc. In our calculation, A tetraquark state with diquarkanti-diquark structure can describe $Y(2175)$ very well. (For comparison, the state in the naive quark model and chiral quark model has much higher mass, $2422 \mathrm{MeV}$ in the naive quark model and 2387 $\mathrm{MeV}$ in the chiral quark model.) The mass of the corresponding scalar state $I^{G} J^{P C}=0^{+} 0^{++}$of $s s \bar{s} \bar{s}$ is calculated to be $1925 \mathrm{MeV}$, which is a little lower than the mass of scalar meson $f_{0}(2020)$.

By calculation the distance between quarks, we can get the spacial structure of the tetraquark. For state $Y(2175)$, the results are

$$
\begin{gathered}
\left\langle\mathbf{r}_{12}^{2}\right\rangle=\left\langle\mathbf{r}_{34}^{2}\right\rangle=1.0 \mathrm{fm}^{2} \\
\left\langle\mathbf{r}_{13}^{2}\right\rangle=\left\langle\mathbf{r}_{14}^{2}\right\rangle=\left\langle\mathbf{r}_{23}^{2}\right\rangle=\left\langle\mathbf{r}_{24}^{2}\right\rangle=1.5 \mathrm{fm}^{2}
\end{gathered}
$$

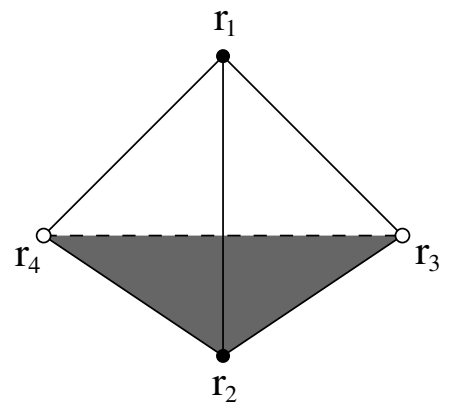

Fig. 2. Spacial structure of the $Y(2175)$ 
Obviously, the $Y(2175)$ can not be planar, as shown in the Fig. 2.

From Table 3, we can see an interesting phenomenon, Taking as the possible candidates of scaler meson observed experimentally, the ground states contain $s, c$ quarks in our model have energies about $100 \mathrm{MeV}$ lower than experiment values, while the excited states are consistent with experiment values. Further study is needed to clarify the situation. The reason may be that the coupling to two-quark state is missing, which the coupling will push the tetraquark state higher.

\section{Unquenched Quark Model for Charmonium}

To unify the description of meson, the unquenched quark model should be developed, i.e, the high Fock states, $q^{2} \bar{q}^{2}, q \bar{q} g, q^{3} \bar{q}^{3}, g g, \cdots$ should be coupled with $q \bar{q}$ to give the meson spectrum. As a first step, we only incorporate the four-quark components in the present work. The meson state reads

$$
|\Psi\rangle=a_{0}\left|\psi_{0}\right\rangle+\sum_{B C} \int c_{B C}(\mathbf{p})|B C ; \mathbf{p}\rangle d^{3} p,
$$

where $\left|\psi_{0}\right\rangle$ represents two-quark state, and $|B C ; \mathbf{p}\rangle$ represents four-quark states, which appear as two mesons with relative motion momentum $\mathbf{p}$. The meson wavefunction satisfies the following Schrödinger equation

$$
H|\Psi\rangle=M|\Psi\rangle,
$$

The system hamiltonian is

$$
H=H_{0}+H_{B C}+H_{I} .
$$

where $H_{0}$ is the hamiltonian for two quark state, which acts on $\psi_{0}$ only,

$$
H_{0}\left|\psi_{0}\right\rangle=M_{0}\left|\psi_{0}\right\rangle,
$$

$H_{B C}$ stands for the four-quark hamiltonian, which acts on $|B C ; \mathbf{p}\rangle$ only.

$$
\begin{gathered}
H_{B C}|B C, \mathbf{p}\rangle=E_{B C}(\mathbf{p})|B C, \mathbf{p}\rangle, \\
E_{B C}\left(\mathbf{P}_{\mathbf{B}}, \mathbf{P}_{\mathbf{C}}\right)=\sqrt{M_{B}^{2}+\mathbf{P}_{B}^{2}}+\sqrt{M_{C}^{2}+\mathbf{P}_{C}^{2}},
\end{gathered}
$$

$H_{I}$ couples two-quark state $\left|\psi_{0}\right\rangle$ and four-quark state $|B C\rangle$. By solving the above Schrödinger equation, the mass of meson can be put as

$$
M=M_{0}+\Delta M,
$$

where $\Delta M$ is the mass shift due to the channel coupling,

$$
\Delta M=\sum_{B C} \int d^{3} p \frac{\left|\left\langle B C\left|H_{I}\right| \psi_{0}\right\rangle\right|^{2}}{M-E_{B C}+i \epsilon} .
$$

For the given channel $B C$, the mass shift

$$
\begin{aligned}
\Delta M_{A}^{B C} & =-\int d^{3} p \frac{\left|\left\langle B C\left|H_{I}\right| \psi_{0}\right\rangle\right|^{2}}{E_{B C}-M+i \epsilon} \\
& =-\int d^{3} p \frac{\left|\mathcal{M}^{J L}\right|^{2}}{\sqrt{M_{B}^{2}+p^{2}}+\sqrt{M_{C}^{2}+p^{2}}-M},
\end{aligned}
$$

The percentage of two-quark state in the physical state of meson can also be obtained,

$$
N^{2}=\left[1+\sum_{B C} \sum_{J L} \int d^{3} p \frac{\left|M^{J L}\right|^{2}}{\left(\sqrt{M_{B}^{2}+p^{2}}+\sqrt{M_{C}^{2}+p^{2}}-M\right)^{2}}\right]^{-1}
$$




\section{EPJ Web of Conferences}

To calculate the transition amplitude $\mathcal{M}^{J L}$, the ${ }^{3} P_{0}$ model, also called quark-pair creation model, which is developed to account for the strong decay of hadron [24], is used. In the model, it assumes that quark-antiquark pair are created with vacuum quantum number $J^{P C}=0^{++}$. The diagrams of all possible decay process $A \rightarrow B+C$ of meson are shown in Fig.3. In many cases only one of them contributes to the strong decay of meson. The transition operator in the model takes the form,
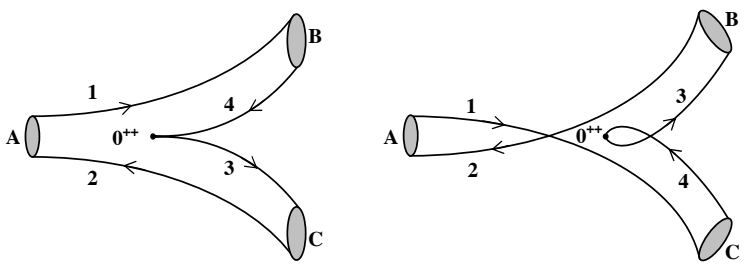

Fig.3 The two possible diagrams contributing to $A \rightarrow B+C$ in the ${ }^{3} P_{0}$ model.

$$
H_{I}=-3 \gamma \sum_{m}\langle 1 m 1-m \mid 00\rangle \int d \mathbf{p}_{3} d \mathbf{p}_{4} \delta^{3}\left(\mathbf{p}_{3}+\mathbf{p}_{4}\right) \mathcal{Y}_{1}^{m}\left(\frac{\mathbf{p}_{3}-\mathbf{p}_{4}}{2}\right) \chi_{1-m}^{34} \phi_{0}^{34} \omega_{0}^{34} b_{3}^{\dagger}\left(\mathbf{p}_{3}\right) d_{4}^{\dagger}\left(\mathbf{p}_{4}\right),
$$

where $\gamma$, which is a dimensionless parameter, represents the probability of the quark-antiquark pair created from the vacuum and can be determined by fitting the observed strong decay of hadrons. $\phi_{0}^{34}=(u \bar{u}+d \bar{d}+s \bar{s}) / \sqrt{3}, \quad \omega_{0}^{34}=(r \bar{r}+g \bar{g}+b \bar{b}) / \sqrt{3}$ are flavor and color singlet state, respectively. $\chi_{1,-m}^{34}$ is a spin-triplet state. $\mathcal{Y}_{l}^{m}(\mathbf{p}) \equiv|p|^{l} Y_{l}^{m}\left(\theta_{p}, \phi_{p}\right)$ is the $l$ th solid harmonic polynomial that reflects the momentum-space distribution of the created quark-antiquark pair. $b_{3}^{\dagger}\left(\mathbf{p}_{3}\right), d_{4}^{\dagger}\left(\mathbf{p}_{4}\right)$ are the creation operators of the quark and antiquark, respectively.

In general, the mock state is adopted to describe the meson with the spatial wave function $\psi_{n_{A} L_{A} M_{L_{A}}}$ $\left(\mathbf{p}_{1}, \mathbf{p}_{2}\right)$ in the momentum representation [25].

$$
\begin{aligned}
\left|A\left(n_{A}{ }^{2 S_{A}+1} L_{A} J_{A} M_{J_{A}}\right)\left(\mathbf{P}_{A}\right)\right\rangle \equiv & \sqrt{2 E_{A}} \sum_{M_{L_{A}}, M_{S_{A}}}\left\langle L_{A} M_{L_{A}} S_{A} M_{S_{A}} \mid J_{A} M_{J_{A}}\right\rangle \\
& \times \int d \mathbf{p}_{1} d \mathbf{p}_{2} \psi_{n_{A} L_{A} M_{L_{A}}}\left(\mathbf{p}_{1}, \mathbf{p}_{2}\right) \chi_{S_{A} M_{S_{A}}}^{12} \phi_{A}^{12} \omega_{A}^{12}\left|q_{1}\left(\mathbf{p}_{1}\right) \bar{q}_{2}\left(\mathbf{p}_{2}\right)\right\rangle
\end{aligned}
$$

with the normalization conditions

$$
\left\langle A\left(n_{A}{ }^{2 S_{A}+1} L_{A} J_{A} M_{J_{A}}\right)\left(\mathbf{P}_{A}\right) \mid A\left(n_{A}{ }^{2 S_{A}+1} L_{A} J_{A} M_{J_{A}}\right)\left(\mathbf{P}_{A}^{\prime}\right)\right\rangle=2 E_{A} \delta^{3}\left(\mathbf{P}_{A}-\mathbf{P}_{A}^{\prime}\right) .
$$

where $n_{A}$ represent the radial quantum number of the meson $A$ composed of $q_{1}, \bar{q}_{2}$ with momentum $\mathbf{p}_{\mathbf{1}}$ and $\mathbf{p}_{\mathbf{2}} . E_{A}$ is the total energy and $\mathbf{P}_{A}$ is the momentum of the meson $A$. $\mathbf{S}_{A}=\mathbf{s}_{q_{1}}+\mathbf{s}_{q_{2}}, \mathbf{J}_{A}=$ $\mathbf{L}_{A}+\mathbf{S}_{A}$ stand for the total spin and total angular momentum, respectively. $\mathbf{L}_{A}$ is the relative orbital angular momentum between $q_{1}$ and $\bar{q}_{2} .\left\langle L_{A} M_{L_{A}} S_{A} M_{S_{A}} \mid J_{A} M_{J_{A}}\right\rangle$ denotes a Clebsch-Gordan coefficient, and $\chi_{S_{A} M_{S_{A}}}^{12}, \phi_{A}^{12}$ and $\omega_{A}^{12}$ are the spin, flavor and color wave functions, respectively.

By using the above transition operator and the meson wave functions, The transition matrix element can be calculated,

$$
\left\langle B C\left|H_{I}\right| A\right\rangle=\delta^{3}\left(\mathbf{P}_{A}-\mathbf{P}_{B}-\mathbf{P}_{C}\right) \mathcal{M}^{M_{J_{A}} M_{J_{B}} M_{J_{C}}},
$$

where $\mathcal{M}^{M_{J_{A}} M_{J_{B}} M_{J_{C}}}$ is the helicity amplitude of $A \rightarrow B+C$. In the center of mass frame of meson $A$, $\mathbf{P}_{A}=0$, and $\mathcal{M}^{M_{J_{A}} M_{J_{B}} M_{J_{C}}}$ can be written as (color factor gives $1 / 3$ )

$$
\begin{aligned}
& \mathcal{M}^{M_{J_{A}} M_{J_{B}} M_{J_{C}}}(\mathbf{P})=\gamma \sqrt{8 E_{A} E_{B} E_{C}} \sum_{\text {all } m \text { 's }}\left\langle L_{A} M_{L_{A}} S_{A} M_{S_{A}} \mid J_{A} M_{J_{A}}\right\rangle\left\langle L_{B} M_{L_{B}} S_{B} M_{S_{B}} \mid J_{B} M_{J_{B}}\right\rangle \\
& \times\left\langle L_{C} M_{L_{C}} S_{C} M_{S_{C}} \mid J_{C} M_{J_{C}}\right\rangle\langle 1 m 1-m \mid 00\rangle\left\langle\chi_{S_{B} M_{S_{B}}}^{14} \chi_{S_{C} M_{S_{C}}}^{32} \mid \chi_{S_{A} M_{S_{A}}}^{12} \chi_{1-m}^{34}\right\rangle\left[\left\langle\phi_{B}^{14} \phi_{C}^{32} \mid \phi_{A}^{12} \phi_{0}^{34}\right\rangle\right. \\
& \left.\times \mathcal{I}_{M_{L_{B}}, M_{L_{C}}}^{M_{L_{A}}, m}\left(\mathbf{P}, m_{1}, m_{2}, m_{3}\right)+(-1)^{1+S_{A}+S_{B}+S_{C}}\left\langle\phi_{B}^{32} \phi_{C}^{14} \mid \phi_{A}^{12} \phi_{0}^{34}\right\rangle \mathcal{I}_{M_{L_{B}}, M_{L_{C}}}^{M_{L_{A}}, m}\left(-\mathbf{P}, m_{2}, m_{1}, m_{3}\right)\right],
\end{aligned}
$$


with the momentum space integral,

$$
\mathcal{I}_{M_{L_{B}}, M_{L_{C}}}^{M_{L_{A}}, m}=\int d \mathbf{p} \psi_{n_{B} L_{B} M_{L_{B}}}^{*}\left(\frac{m_{3}}{m_{1}+m_{3}} \mathbf{P}+\mathbf{p}\right) \psi_{n_{C} L_{C} M_{L_{C}}}^{*}\left(\frac{m_{3}}{m_{2}+m_{3}} \mathbf{P}+\mathbf{p}\right) \psi_{n_{A} L_{A} M_{L_{A}}}(\mathbf{P}+\mathbf{p}) \mathcal{Y}_{1}^{m}(\mathbf{p}),
$$

where $\mathbf{P}=\mathbf{P}_{B}=-\mathbf{P}_{C}, \mathbf{p}=\mathbf{p}_{3}, m_{3}$ is the mass of the created quark $q_{3} ;\left\langle\chi_{S_{B} M_{S_{B}}}^{14} \chi_{S_{C} M_{S_{C}}}^{32} \chi_{S_{A} M_{S_{A}}}^{12} \chi_{1-m}^{34}\right\rangle$ and $\left\langle\phi_{B}^{14} \phi_{C}^{32} \mid \phi_{A}^{12} \phi_{0}^{34}\right\rangle$ are the overlap of spin and flavor wave functions, respectively.

The flavor overlap can be evaluated directly and the spin overlap can be obtained in terms of Winger's $9 j$ symbol,

$$
\begin{aligned}
\left\langle\chi_{S_{B} M_{S_{B}}}^{14} \chi_{S_{C} M_{S_{C}}}^{32} \chi_{S_{A} M_{S_{A}}}^{12} \chi_{1-m}^{34}\right\rangle= & \sum_{S, M_{S}}\left\langle S_{B} M_{S_{B}} S_{C} M_{S_{C}} \mid S M_{S}\right\rangle\left\langle S_{A} M_{S_{A}} 1-m \mid S M_{S}\right\rangle \\
& \times(-1)^{S_{C}+1} \sqrt{3\left(2 S_{A}+1\right)\left(2 S_{B}+1\right)\left(2 S_{C}+1\right)}\left\{\begin{array}{ccc}
\frac{1}{2} & \frac{1}{2} & S_{A} \\
\frac{1}{2} & \frac{1}{2} & 1 \\
S_{B} & S_{C} & S
\end{array}\right\} .
\end{aligned}
$$

To evaluate the momentum space integral, the space wave function must be fixed. Generally, one takes the simple harmonic oscillator (SHO) approximation for the meson space wave functions. It reads

$$
\Psi_{n L M_{L}}(\mathbf{p})=(-1)^{n}(-i)^{L} R^{L+\frac{3}{2}} \sqrt{\frac{2 n !}{\Gamma\left(n+L+\frac{3}{2}\right)}} \exp \left(-\frac{R^{2} p^{2}}{2}\right) L_{n}^{L+\frac{1}{2}}\left(R^{2} p^{2}\right) y_{L M_{L}}(\mathbf{p}),
$$

with $\mathcal{Y}_{L M_{L}}(\mathbf{p})=|\mathbf{p}|^{L} Y_{L M_{L}}\left(\Omega_{p}\right)$. Here $R$ denotes the SHO wave function scale parameter, which is fixed by fitting the wave function obtained by solving Schödinger equation of the corresponding meson; $\mathbf{p}$ represents the relative momentum between the quark and the antiquark within a meson; $L_{n}^{L+\frac{1}{2}}\left(R^{2} p^{2}\right)$ is an associated Laguerre polynomial.

The partial-wave amplitude in Eq.(41) can be related to the helicity amplitude in Eq.(47) via the Jacob-Wick formula [26]

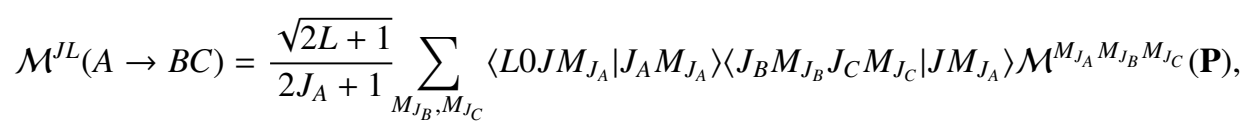

where $\mathbf{J}=\mathbf{J}_{B}+\mathbf{J}_{C}, \mathbf{J}_{A}=\mathbf{J}_{B}+\mathbf{J}_{C}+\mathbf{L}$, and $M_{J_{A}}=M_{J_{B}}+M_{J_{C}}$.

Combining Eqs.(41), (49) and (51), the mass shift can be obtained. To be noted that the physical mass $M$ also appears at the right-hand side of Eq.(41), the iteration method should be used here. First by setting $M=M_{A}$, the mass shift $\Delta M$ and the new physical mass are obtained, then substituting new physical mass in the Eq.(41), the new mass shift is obtained. Continue this process until a stable physical mass is reached.

Recently the mass shifts for charmonium, bottomonium and other mesons have been calculated by several groups [27]. The results show that the mass shifts are rather large, especially for the light mesons. So the contribution from the four-quark components cannot be ignored. Of course, part of the effects can be absorbed by the model parameters. It is very interesting to see that can the unquenched quark model improve the agreement between theoretical results and the experimental data? In the following, we take charmonium as an example, adjusting the model parameters and re-fitting the experimental data by incorporating the four-quark components in the quark model.

Table 4. The re-adjusted parameters.

\begin{tabular}{ccccc}
\hline$m_{u}=m_{d}(\mathrm{MeV})$ & $m_{s}(\mathrm{MeV})$ & $m_{c}(\mathrm{MeV})$ & $\alpha_{s}(c \bar{c})$ & $\alpha_{s}(c \bar{q})$ \\
321 & 587 & 1758 & 0.40 & 0.58 \\
\hline
\end{tabular}

The model parameters of the unquenched quark model for charmonium is listed in Table 4. As a preliminary study, only the masses of quarks and the coupling constant $\alpha_{s}$ of OGE are re-adjusted, other parameters keep their value in the model without high Fock components. $\gamma=6.95$ and $\gamma_{s}=$ $\gamma / \sqrt{3}$ [28]. The calculated charmonium spectrum is shown in Table 5. 
EPJ Web of Conferences

Table 5. The masses of charmonium $c \bar{c}$ (unit: $\mathrm{MeV}$ ).

\begin{tabular}{|c|c|c|c|c|c|c|}
\hline$n^{2 S+1} L_{J}$ & $\beta(\mathrm{GeV})$ & $c \bar{c}$ mass & mass shift & physical mass & exp. mass & $c \bar{c}$ ratio $(\%)$ \\
\hline${ }^{1} S_{0}$ & 0.730 & 3163 & -184 & 2979 & $2980.3 \pm 1.2$ & 92.75 \\
\hline $2^{1} S_{0}$ & 0.560 & 3850 & -216 & 3634 & $3637 \pm 4$ & 88.90 \\
\hline $3{ }^{1} S_{0}$ & 0.478 & 4250 & -190 & 4060 & & 87.99 \\
\hline $4^{1} S_{0}$ & $0.390-0.446$ & 4527 & $-140-165$ & $4387-4362$ & & $89.62-87.74$ \\
\hline $5^{1} S_{0}$ & $0.360-0.390$ & 4727 & $-123-131$ & $4604-4596$ & & - \\
\hline $1^{3} S_{1}$ & 0.632 & 3295 & -186 & 3109 & $3096.916 \pm 0.011$ & 91.80 \\
\hline $2^{3} S_{1}$ & 0.504 & 3918 & -208 & 3710 & $3686.09 \pm 0.04$ & 88.16 \\
\hline $3^{3} S_{1}$ & 0.446 & 4296 & -181 & 4115 & & 87.62 \\
\hline $4^{3} S_{1}$ & $0.366-0.424$ & 4560 & $-135-159$ & $4425-4401$ & & - \\
\hline $5^{3} S_{1}$ & $0.348-0.374$ & 4751 & $-121-128$ & $4630-4623$ & & - \\
\hline $1^{1} P_{1}$ & 0.528 & 3723 & -207 & 3516 & $3525.93 \pm 0.27$ & 88.93 \\
\hline $2^{1} P_{1}$ & 0.462 & 4157 & -194 & 3963 & & 87.30 \\
\hline $3^{1} P_{1}$ & 0.398 & 4457 & -151 & 4306 & & 88.27 \\
\hline $4^{1} P_{1}$ & $0.366-0.398$ & 4674 & $-129-140$ & $4545-4534$ & & - \\
\hline $5^{1} P_{1}$ & $0.332-0.366$ & 4833 & $-112-118$ & $4721-4715$ & & - \\
\hline $1^{3} P_{0}$ & 0.526 & 3662 & -199 & 3463 & $3414.75 \pm 0.31$ & 89.58 \\
\hline $2^{3} P_{0}$ & 0.460 & 4114 & -187 & 3927 & & 87.93 \\
\hline $3^{3} P_{0}$ & 0.396 & 4425 & -147 & 4278 & & 88.81 \\
\hline $4^{3} P_{0}$ & $0.366-0.398$ & 4654 & $-126-138$ & $4528-4517$ & & - \\
\hline $5^{3} P_{0}$ & $0.332-0.366$ & 4815 & $-111-116$ & 4704-4699 & & - \\
\hline $1^{3} P_{1}$ & 0.526 & 3693 & -202 & 3491 & $3510.66 \pm 0.07$ & 89.31 \\
\hline $2^{3} P_{1}$ & 0.460 & 4137 & -190 & 3947 & & 87.67 \\
\hline $3^{3} P_{1}$ & 0.396 & 4442 & -148 & 4294 & & 88.65 \\
\hline $4^{3} P_{1}$ & $0.366-0.39$ & 4665 & $-127-136$ & $4538-4529$ & & - \\
\hline $5^{3} P_{1}$ & $0.332-0.366$ & 4824 & $-111-117$ & 4713-4707 & & - \\
\hline $1^{3} P_{2}$ & 0.526 & 3757 & -213 & 3544 & $3556.93 \pm 0.27$ & 88.42 \\
\hline $2^{3} P_{2}$ & 0.460 & 4181 & -197 & 3984 & & 86.83 \\
\hline $3^{3} P_{2}$ & 0.396 & 4476 & -154 & 4322 & & 87.77 \\
\hline $4^{3} P_{2}$ & $0.366-0.39$ & 4687 & $-131-143$ & $4556-4544$ & & - \\
\hline $5^{3} P_{2}$ & $0.332-0.366$ & 4844 & $-114-120$ & $4730-4724$ & & - \\
\hline $1^{1} D_{2}$ & 0.462 & 4027 & -200 & 3827 & & 86.91 \\
\hline $2^{1} D_{2}$ & 0.418 & 4361 & -167 & 4194 & & 86.94 \\
\hline $3^{1} D_{2}$ & 0.376 & 4603 & -134 & 4469 & & 87.57 \\
\hline $4^{1} D_{2}$ & $0.336-0.376$ & 4781 & $-113-121$ & $4668-4660$ & & - \\
\hline $5^{1} D_{2}$ & $0.296-0.336$ & 4910 & $-102-105$ & $4808-4805$ & & - \\
\hline $1^{3} D_{1}$ & 0.462 & 4003 & -197 & 3806 & & 87.23 \\
\hline $2^{3} D_{1}$ & 0.418 & 4343 & -165 & 4178 & & 87.16 \\
\hline $3^{3} D_{1}$ & 0.376 & 4590 & -132 & 4458 & & - \\
\hline $4^{3} D_{1}$ & $0.336-0.376$ & 4770 & $-111-121$ & $4659-4649$ & & - \\
\hline $5^{3} D_{1}$ & $0.296-0.336$ & 4902 & $-102-104$ & $4800-4798$ & & - \\
\hline $1^{3} D_{2}$ & 0.462 & 4019 & -199 & 3820 & & 87.06 \\
\hline $2^{3} D_{2}$ & 0.418 & 4355 & -166 & 4189 & & 87.06 \\
\hline $3^{3} D_{2}$ & 0.376 & 4599 & -134 & 4465 & & 87.59 \\
\hline $4^{3} D_{2}$ & $0.336-0.376$ & 4777 & $-112-121$ & $4665-4656$ & & - \\
\hline $5^{3} D_{2}$ & $0.296-0.336$ & 4907 & $-101-104$ & $4806-4803$ & & - \\
\hline $1^{3} D_{3}$ & 0.462 & 4043 & -204 & 3839 & & 86.56 \\
\hline $2^{3} D_{3}$ & 0.418 & 4373 & -170 & 4203 & & 86.63 \\
\hline $3^{3} D_{3}$ & 0.376 & 4614 & -137 & 4477 & & - \\
\hline $4^{3} D_{3}$ & $0.336-0.376$ & 4788 & $-114-123$ & $4674-4665$ & & - \\
\hline $5^{3} D_{3}$ & $0.296-0.336$ & 4915 & $-103-106$ & $4812-4809$ & & - \\
\hline
\end{tabular}


There are several features of our calculation:

(1) From Table 5, we can see the percentages of $c \bar{c}$ in the physical states are all around $90 \%$, which are different from the results of the previous work (where the percentage may be as low as $50 \%$ in $P$ wave states). The high percentage of $c \bar{c}$ is a welcome property, which make our calculation consistent, the higher Fock states, $q^{3} \bar{q}^{3}, \cdots$ can be neglected safely.

(2) The mass shifts for the states with the same $n, L$ are almost same, which is consistent with other calculation $[27,29]$.

(3) The experimental masses below the $D \bar{D}$ threshold are reproduced well, similar to the results of the quenched quark model (only $c \bar{c}$ component). This means that for the low-lying states, the fourquark contributions can be totally absorbed into the parameters. For the states $\psi(3770), \psi(4040)$, $\psi(4160)$ and $\psi(4415)$, the model gives the masses close to experimental data, if these states are taken as $1^{3} D_{1}, 3^{3} S_{1}, 2^{3} D_{1}$ and $4^{3} S_{1}$.

(4) For given $J, L$, The mass shifts for small $n$ is larger than that for large $n$. This feature leads to that the high states will be pushed higher, which it cannot be absorbed into parameters of the quenched quark model.

(5) As for the new charmonia ( $X Y Z$ particles) observed in recent years [2], some states can be identified as states in Table 5, some states cannot be allocated due to their possible exotic structures, tetraquark, glueball, molecule, hybrid, etc. [2]. The assignments are listed in Table 6. To keep in mind, these assignments are based on the masses only. To validate the assignments, decay properties calculation of these states needs to be done.

Table 6. The assignments of $X Y Z$ states.

\begin{tabular}{c|ccccc}
\hline states & $X(3872)$ & $X(3915)$ & $Z(3930)$ & $X(3940)$ & $Y(3940)$ \\
\hline assignment & $1^{1} D_{2}$ & $2^{3} P_{0}$ & $2^{3} P_{2}$ & $2^{3} P_{1}$ & $2^{1} P_{1}$ \\
\hline mass $(\mathrm{MeV})$ & 3827 & 3927 & 3984 & 3947 & 3963 \\
\hline states & $X(4140)$ & $X(4160)$ & $X(4350)$ & $Y(4630)$ & $Y(4660)$ \\
\hline assignment & $2^{1} D_{2}$ & $2^{1} D_{2}$ & $3^{3} P_{2}$ & $5^{3} S_{1}$ & $4^{3} D_{1}$ \\
\hline mass $(\mathrm{MeV})$ & 4194 & 4194 & 4322 & 4630 & 4659 \\
\hline
\end{tabular}

\section{Summary}

The non-relativistic quark model has achieved great success in describing the properties of hadrons, especially for the low-lying states. With advance of experiments, more excited states are observed, the quark model should be developed. Unquenching thequark model is an important step for the development. How to unquench the quark model is still an open question. In the present work, the commonly used ${ }^{3} P_{0}$ model is employed and the unquenched quark model is applied to explore the charmonium spectrum. The calculation shows that the effects of including the four-quark component will affect the masses of highly excited states while keeping the agreement between theoretical results and experimental data for the low-lying states. The calculation also shows that the higher Fock states, $q^{3} \bar{q}^{3}$ can be neglected safely. The further work on unquenching quark model is to improve the wave functions of created quark-pair, the approximation of plane wave is used at the moment.

Another development of quark model is to study the multi-quark system, where the multi-body interaction may play an important role. Based on the lattice QCD calculation, a string (flux-tube) model is proposed to study the tetraquark system. The calculated results are encouraging, most of the scalar mesons can be described as tetraquark states in the model. An important feature of the model is that an alternative form of color confinement is used. So the multi-quark system is a good place to check the validity of the Casimir scaling [16].

Another problem to be solved is how to coupling the Fock states with different color structures. String model is a good starting point. The mechanism to break and re-join the string needs to clarify.

\section{Acknowledgment}

This work is supported partly by the National Science Foundation of China under Contract Nos. 11035006, 10775072, 11047140 and 10947160, the Research Fund for the Doctoral Program of Higher 
EPJ Web of Conferences

Education of China under Grant No. 20070319007 and the PhD Program Funds of Chongqing Jiaotong University.

\section{References}

1. C. R. Deng, J. L. Ping, F. Wang and T. Goldman, Phys. Rev. D82 (2010) 074001.

2. N. Brambilla, S. Eidelman, B. K. Heltsley, et al, Eur. Phys. J. C71 (2011) 1534 and references therein.

3. A. Manohar and H. Georgi, Nucl. Phys. B234 (1984) 189.

4. J. Vijande, F. Fernandez and A. Valcarce, J. Phys. G31 (2005) 481.

5. L. Z. Chen, H. R. Pang, H. X. Huang, J. L. Ping and F. Wang, Phys. Rev. C76 (2007) 014001.

6. R. K. Bhaduri, L. E. Cohler and Y. Nogami, Phys. Rev. Lett. 44 (1980) 1369.

7. J. Weinstein and N. Isgur, Phys. Rev. D27 (1983) 588.

8. E. Hiyama, Y. Kino and M. Kamimura, Prog. Part. Nucl. Phys. 51 (2003) 223.

9. L. Wang, J. L. Ping, Chin. Phys. Lett. 24 (2007) 1195.

10. T. T. Takahashi, H. Matsufuru, Y. Nemoto and H. Suganuma, Phys. Rev. Lett. 86 (2001) 18; T. T. Takahashi, H. Suganuma, Y. Nemoto and H. Matsufuru, Phys. Rev. D65 (2002) 114509; F. Okiharu, H. Suganuma and T. T. Takahashi, Phys. Rev. Lett. 94 (2005) 192001.

11. L. Maiani, F. Piccinini, A. D. Polosa et al, Phys. Rev. Lett. 93 (2004) 21.

12. L. Maiani, V. Riquer, F. Piccinini and A.D. Polosa, Phys. Rev. D72 (2005) 031502.

13. M. Iwasaki and T. Fukutome Phys. Rev. D72 (2005) 094016.

14. G. J. Ding and M. L. Yan, Phys. Lett. B643 (2006) 33.

15. K. Johnson and C.B. Thorn, Phys. Rev. D13 (1976) 1934; C. Semay, Eur. Phys. J. A22 (2004) 353.

16. G. S. Bali, Phys. Rev. D62 (2000) 114503.

17. H. X. Chen, A. Hosaka and S. L. Zhu, Phys. Rev. D76 (2007) 094025.

18. B. Aubert et al [BABAR Collaboration], Phys. Rev. D74 (2006) 091103; Phys. Rev. D76 (2007) 012008 ; D77 (2008) 092002.

19. M. Ablikim et al [BES Collaboration], Phys. Rev. Lett. 100 (2008) 102003.

20. G. J. Ding and M. L. Yan, Phys. Lett. B650 (2007) 390; B657 (2007) 49.

21. Z. G. Wang, Nucl. Phys. A791 (2007) 106.

22. S. L. Zhu, Int. J. Mod. Phys. E17 (2008) 283.

23. A. M. Torres, K. P. Khemchandani, L. S. Geng et al, Phys. Rev. D78 (2008) 074031.

24. L. Micu, Nucl. Phys. B10 (1969) 521; A.Le Yaouanc, L. Oliver, O. Pene and J-C. Raynal, Phys. Rev. D8 (1973) 2223; 9(1974) 1415; 11 (1975) 1272; W. Roberts and B. Silvestr-Brac, Few-Body Syst. 11 (1992) 171; E. S. Ackleh, T. Barnes and E. S. Swanson, Phys. Rev. D54 (1996) 6811; T. Barnes, S. Godfrey and E. S. Swanson, Phys. Rev. D72 (2005) 054026.

25. C. Hayne and N. Isgur, Phys. Rev. D25 (1982) 1944.

26. M. Jacob and G. C. Wick, Ann. Phys. (Leipzig) 7 (1959) 404; Ann. Phys. (N.Y.) 281 (2000) 774.

27. T. Barnes and E. S. Swanson, Phys. Rev. C77 (2008) 055206; Yu. S. Kalashinikova, Phys. Rev. D72 (2005) 034010; B. Q. Li, C. Meng and K. T. Chao, Phys. Rev. D80 (2009) 014012.

28. H. G. Blundell and S. Godfrey, Phys. Rev. D53 (1996) 3700; D53 (1996) 3712; J. Lu, W. Z. Deng, X. L. Chen and S. L. Zhu, Phys. Rev. D73 (2006) 054012.

29. F. E. Close and C. E. Thomas, Phys. Rev. C79 (2009) 045201. 